\title{
Desarrollo de intermetálicos TiAl mediante técnicas pulvimetalúrgicas convencionales y de alta densificación
}

\author{
TiAl Intermetallic Development by Using Conventional and High \\ Densification Pouder Metallurgy Techniques
}

Fecha de Recepción: 22 de Diciembre de 2013

Fecha de Aprobación: 24 de Enero de 2014

\author{
Vicente Amigó-Borrás* \\ Angélica Amigó-Mata** \\ Montserrat Haro-Rodríguez ${ }^{* \star *}$
}

\section{Resumen}

Cada día hay más demanda de materiales que ofrezcan altas temperaturas de servicio y bajo peso; pero su fabricación es compleja y costosa, particularmente la de las superaleaciones de base cobalto y la de las aleaciones de titanio; dentro de estas últimas, los intermetálicos TiAl y $\mathrm{Ti}_{3} \mathrm{Al}$ son ampliamente reconocidos para satisfacer las necesidades actuales; sin embargo, la colada y forja de estos intermetálicos, que tienen una mejor resistencia frente a la oxidación a elevadas temperaturas, resulta muy compleja, y es por lo que, partiendo de polvo prealeado, se busca obtener productos prácticamente acabados con un coste razonable. El presente trabajo analiza la influencia de las variables de procesado de polvos intermetálicos Ti48A12Cr2 $\mathrm{Nb}$, mediante técnicas pulvimetalúrgicas convencionales y Spark Plasma Sintering (SPS), en su microestructura y en sus

\begin{abstract}
There is a growing demand for materials that offer services at high temperatures and low weight. However, these materials' manufacture is complex and expensive, particularly the cobaltbased superalloys and the titanium alloys. Within the latter, the $\mathrm{Ti}_{3} \mathrm{Al}$ and the TiAl intermetallics are widely recognized to meet the current needs. However, due that these intermetallic casting and forging process, which have a better oxidation resistance at elevated temperatures, it becomes very complex and therefore starting from a prealloyed powder, can be tried to obtain practically some finished products at a reasonable cost. This paper analyzes the Ti48A12Cr2Nb intermetallic powder processing variables' influence, by the conventional powder metallurgy techniques and the Spark Plasma Sintering, (SPS) in their microstructure and mechanical properties.
\end{abstract}

* Ph.D.Universidad Politécnica de Valencia (Valencia, España). vamigo@mcm.upv.es

** M.Sc. Universidad Politécnica de Valencia (Valencia, España). anamma@posgrado.upv.es

*** M.Sc. Universidad Politécnica de Valencia (Valencia, España).monharod@upvnet.upv.es 
propiedades mecánicas. Se obtienen muestras a diferentes temperaturas de sinterización a partir de polvos obtenidos por atomización. La influencia en las propiedades mecánicas se observa mediante su microdureza y resistencia a la compresión, realizándose un seguimiento de la microestructura mediante microscopía óptica y electrónica de barrido.

Las condiciones de procesado muestran un gran efecto en la microestructura obtenida, fundamentalmente en la formación de la fase $\boldsymbol{\alpha}_{2}$, que acompaña las propiedades mecánicas finales. Sin embargo, es con el proceso de máxima densificación donde se obtienen las propiedades adecuadas, lo cual hace pensar que es una alternativa clara a los procesos actuales de colada y deformación plástica.

Palabras clave: Intermetálico TiAl, Pulvimetalurgia, Técnica SPS, Sinterización.
Some samples are obtained at different sintering temperatures from the powders obtained by atomization.

The influence on the mechanical properties is observed by its micro-hardness and compression resistance, and their microstructure is analyzed by an optical and scanning electron microscopy. The processing conditions show a large effect on the obtained microstructure, mainly in the $\boldsymbol{\alpha}_{2}$ phase formation, which accompanies the final mechanical properties. However, is in the maximum densification process, where the appropriate properties are obtained, which suggests that they are a clear alternative to the casting and plastic deformation's current processes.

Keywords: TiAl Intermetallic, Powder Metallurgy, Spark Plasma Sintering, SPS technique, Mechanical properties. 


\section{INTRODUCCIÓN}

Las aleaciones Ti-Al, y especialmente los intermetálicos $\gamma$-TiAl son un reemplazo potencial para las superaleaciones en aplicaciones estructurales de alta temperatura en las industrias aeroespacial y del automóvil, debido a su baja densidad, alta resistencia y rigidez específica, y resistencia a fluencia elevada [1-3]; sin embargo, su desarrollo se ha visto obstaculizado por la falta de tenacidad a temperatura ambiente, su pobre equilibrio de propiedades mecánicas y sus dificultades de fabricación. En estos últimos años se han incrementado notablemente las investigaciones sobre estos materiales y sus procesos de fabricación [4-8].

Los intermetálicos conjugan, de forma lamelar y con mayor o menor intensidad, las fases $\gamma$ y $\boldsymbol{\alpha}_{2}$, correspondientes a los intermetálicos TiAl y $\mathrm{Ti}_{3} \mathrm{Al}$ [9], de modo que las propiedades finales tienen una fuerte dependencia de la distribución entre estas fases [10]; con ello, las propiedades mecánicas dependen principalmente de la orientación lamelar y de variables como el tamaño de grano, su distribución y el espaciado entre las lamelas $\gamma$ y $\boldsymbol{\alpha}_{2}$ [2].

Las adiciones de $\mathrm{Cr}$ y $\mathrm{Nb}$ a los intermetálicos TiAl han demostrado ser beneficiosas para las propiedades mecánicas, dado que mejoran su tenacidad, pues modifican la microestructura de $\gamma$-TiAl a una microestructura dúplex $[4,5]$. El niobio proporciona una buena resistencia frente a la oxidación, pero en proporciones no muy elevadas, mientras que el cromo le proporciona a los aluminiuros una mejora en su deformación plástica a temperatura ambiente [2]. La adición de estos elementos, $\mathrm{Nb}$ y $\mathrm{Cr}$, estabilizan la microestructura de estos intermetálicos como microestructura dúplex, en lugar de la microestructura lamelar, lo que proporciona mayor ductilidad y resistencia a la tracción, especialmente con estructura fina y homogénea; mientras que la microestructura lamelar presenta mayor dureza y resistencia a la fluencia $[5,8]$. Se recurre a la pulvimetalurgia [7] para obtener intermetálicos con tamaño de grano ultrafino [3, 6] y mejorar con ello la ductilidad.

Los intermetálicos se han obtenido generalmente mediante procesos de colada $[3,6]$, pero en los últimos años está tomando mayor auge su obtención por vía pulvimetalúrgica [11-13]. El procesado de polvos mediante prensado y sinterizado no ofrece la adecuada densificación para su uso, por ello se debe recurrir a costosos procesos de consolidación en caliente, como Hot Press (HP) o Hot Isostatic Press (HIP); sin embargo, debido al tiempo de permanencia de las piezas a elevadas temperaturas se produce un fuerte crecimiento de grano que afecta las propiedades generales del material. Por ello, la obtención de estos intermetálicos mediante Spark Plasma Sintering (SPS) presenta unas innegables ventajas, ya que puede controlarse su microestructura al realizarse a temperaturas inferiores y tiempos muy cortos [14].

Como material para conformar los intermetálicos se ha utilizado mezcla elemental de polvos [12, 13], que presenta gran heterogeneidad y bajas propiedades al no obtener las fases adecuadas por la falta de difusión entre los elementos, debido al escaso tiempo y las bajas temperaturas del proceso SPS $[6,15]$. Una alternativa a la mezcla elemental de polvos es la utilización de polvos prealeados atomizados, que permite obtener un gran rango de intermetálicos con excelentes propiedades y densificación prácticamente completa $[14,16$, 17].

En el presente trabajo se estudia la influencia de los parámetros del proceso de sinterización en vacío y por SPS en la microestructura y propiedades del intermetálico Ti48A12Cr2Nb.

\section{Procedimiento eXPerimental}

\section{A. Material}

El polvo utilizado en la investigación es de TiAl, atomizado por gas, suministrado por TLS Technik Spezial Pulver, con la composición indicada en la Tabla 1. 
TABLA 1

COMPOSICIÓN QUíMICA DE LOS POLVOS EMPLEADOS EN LA INVESTIGACIÓN

\begin{tabular}{|c|c|c|c|c|c|c|c|c|}
\hline Elemento & $\mathbf{T i}$ & $\mathbf{C r}(\mathbf{a t} \%)$ & $\mathbf{A l}(\mathbf{a t} \%)$ & $\mathbf{N b}(\mathbf{a t} \%)$ & $\mathbf{F e}(\mathbf{w t} \%)$ & $\mathbf{C}(\mathbf{w t} \%)$ & $\mathbf{N}(\mathbf{w t} \%)$ & $\mathbf{H}(\mathbf{w t} \%)$ \\
\hline & bal. & 1,92 & 47,35 & 2,00 & 0,039 & 0,004 & 0,001 & 0,001 \\
\hline
\end{tabular}

El tamaño de partículas se ha determinado mediante un equipo Mastersizer 2000, de Malvern Instruments, obteniéndose una distribución de tamaño de partícula de $\mathrm{d}(0.1): 12.629 \mathrm{~mm}, \mathrm{~d}(0.5)$ :
$24.950 \mathrm{~mm}$ y d(0.9): $46.523 \mathrm{~mm}$. En la Fig. 1 puede observarse la morfología esférica de los polvos suministrados.

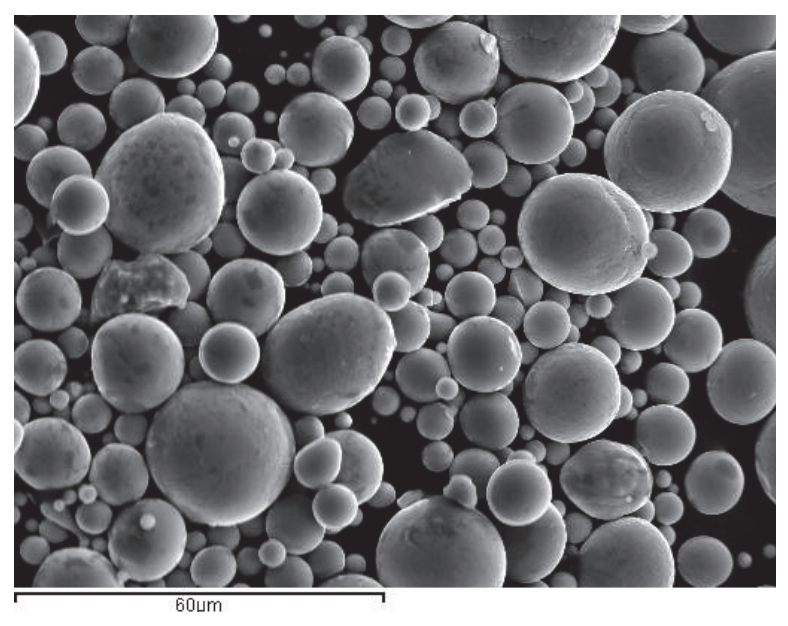

Fig. 1. Imagen electrones secundarios de los polvos atomizados $\mathrm{Ti} 48 \mathrm{Al} 2 \mathrm{Cr} 2 \mathrm{Nb}$.

\section{B. Compactación y sinterización}

Mediante compactación y sinterización se han obtenido probetas para el ensayo de flexión de $32 \times 12 \mathrm{~mm}$. y $5 \mathrm{~mm}$. de espesor; su conformado se ha realizado en matriz flotante con presiones de compactación uniaxiales de $600 \mathrm{MPa}$, utilizando como ligante un adhesivo de metilcelulosa en una proporción del $0,5 \%$ en peso.
La sinterización se realiza en horno de vacío a temperaturas de 1250,1300 y $1400{ }^{\circ} \mathbf{C}$ durante 2 horas (Fig. 2). Debe tenerse en cuenta que a temperaturas inferiores a $1200{ }^{\circ} \mathrm{C}$ se obtienen propiedades mecánicas muy bajas, y que temperaturas superiores a los $1400{ }^{\circ} \mathrm{C}$ provocan un crecimiento de grano importante que merma igualmente sus propiedades mecánicas. 


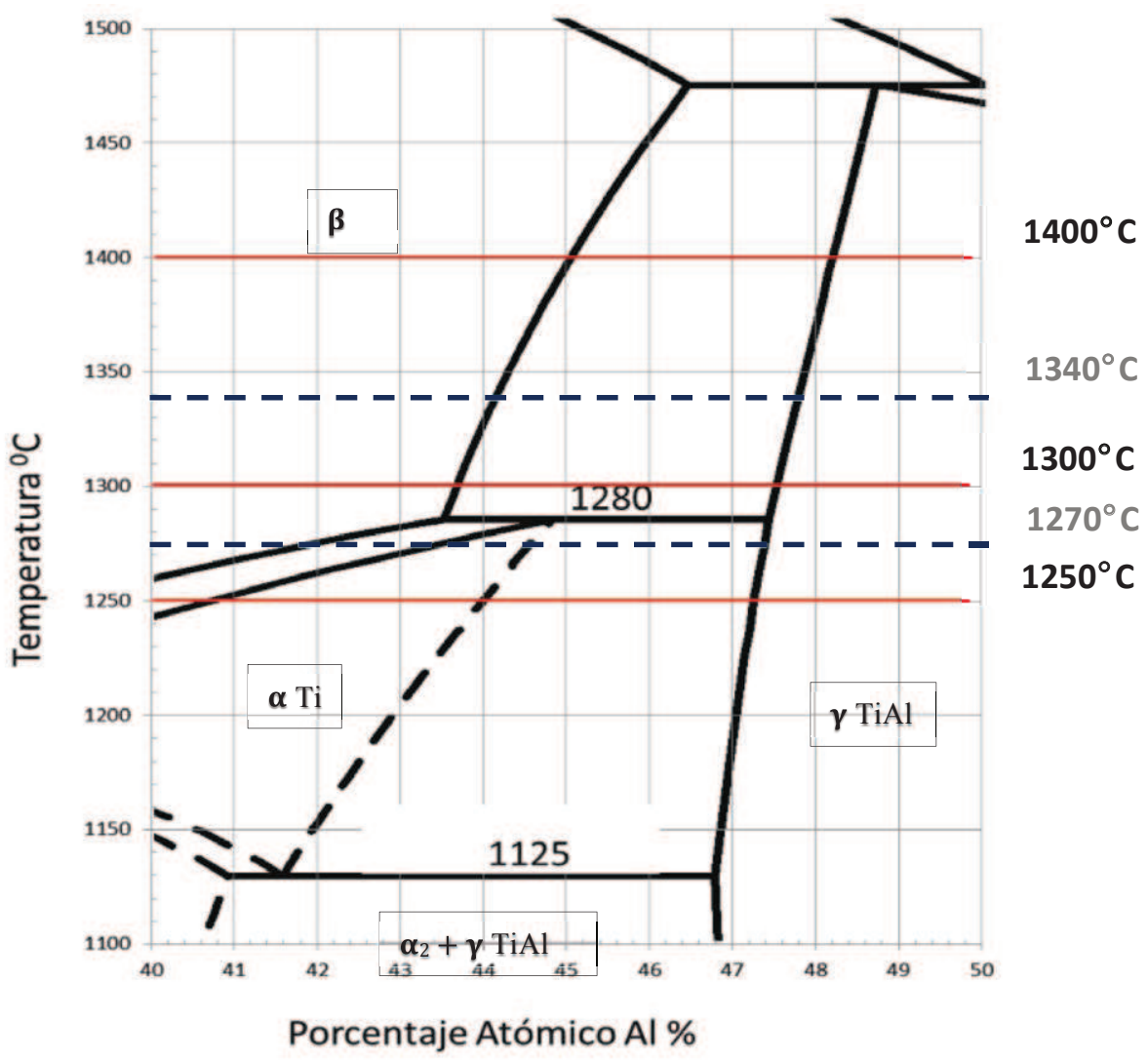

Fig. 2. Detalle del diagrama de equilibrio Ti-Al con indicación de las temperaturas utilizadas; en trazo continuo, proceso de compactación y sinterización; en trazo discontinuo, proceso SPS [18].

La mayor densificación del material se ha realizado en un horno de Spark Plasma Sintering FCT S8451, en las instalaciones de Tecnalia Research \& Innovation. Este equipo puede proporcionar una corriente continua (CC) de $10000 \mathrm{~A}$, con una intensidad máxima de $10 \mathrm{~V}$. La CC se aplica en pulsos de $10 \mathrm{~ms}$ con pausas de $5 \mathrm{~ms}$. El polvo se ha introducido dentro de una cámara donde se mantienen condiciones de vacío, en moldes de grafito de $30 \mathrm{~mm}$ de diámetro, entre dos punzones también de grafito. La temperatura se ha controlado con un pirómetro colocado en el interior del punzón inferior, lo que proporciona una diferencia de temperatura entre el pirómetro y la pieza inferior a $15{ }^{\circ} \mathrm{C}$. Previo al calentamiento, se ha aplicado la presión de compactación de 30 y $50 \mathrm{MPa}$, que es mantenida durante todo el proceso de sinterizado.

La designación de las muestras y los parámetros utilizados en los dos procesos se muestran en la Tabla 2. En la Fig. 2 se indican, en la zona del diagrama de equilibrio de interés, las temperaturas utilizadas en ambos procesos: en líneas continuas, las correspondientes al proceso de compactación y sinterización, y con líneas discontinuas, las temperaturas utilizadas en el proceso SPS. 
TABLA 2

VARIABLES DE LOS PROCESOS DE SINTERIZACIÓN UTILIZADOS EN LA INVESTIGACIÓN

\begin{tabular}{|c|c|c|c|c|}
\hline Muestra & Proceso & Presión $\mathbf{( M P a )}$ & Temperatura $\left.\mathbf{(}^{\mathbf{}} \mathbf{C}\right)$ & Tiempo $(\mathbf{m i n})$. \\
\hline 1-CS & C \& S & 600 & 1250 & 120 \\
\hline 2-CS & C \& S & 600 & 1300 & 120 \\
\hline 3-CS & C \& S & 600 & 1400 & 120 \\
\hline 1-SPS & SPS & 30 & 1270 & 2 \\
\hline 2-SPS & SPS & 50 & 1270 & 2 \\
\hline 3-SPS & SPS & 30 & 1340 & 2 \\
\hline
\end{tabular}

\section{Caracterización de los sinterizados}

El análisis microestructural se ha realizado mediante microscopía óptica (NIKON modelo LV100) y microscopía electrónica de barrido (JEOL JSM 6300), equipado con un espectrómetro de energía dispersiva (EDS Oxford Inst. Ltda.). La densidad se obtiene por el método de Arquímedes, utilizando una balanza analítica Kern modelo 770. La difracción de rayos $\mathrm{X}$ se lleva a cabo utilizando un equipo PANalytical CubiX profesional.

La microdureza se determina mediante el método Vickers en un microdurómetro HMV 2 con carga de 300 g. y un tiempo de aplicación de la carga de $15 \mathrm{~s}$.

El ensayo a flexión se realiza en una Máquina Universal de Ensayos estáticos de $50 \mathrm{KN}$ Instron modelo 4204, según norma UNE-EN ISO-3325. Las muestras ensayadas son rectangulares de 290x110x45 mm, biapoyadas en sus extremos con una distancia entre apoyos de $190 \mathrm{~mm}$. La velocidad de cruceta es de $0.5 \mathrm{~mm} / \mathrm{min}$.

El ensayo a compresión se realiza con una prensa Instron modelo 1343 de $500 \mathrm{KN}$, según norma UNE-EN ISO 7500-1:2006/AC: 2009. Las muestras son cilíndricas de diámetro $10 \mathrm{~mm}$. y altura $10 \mathrm{~mm}$. Se realiza en modo "control de posición” con una velocidad de $0.5 \mathrm{~mm} / \mathrm{min}$.

\section{RESULTADOS Y DISCUSIÓN}

Tras el proceso de sinterización, en ambos procesos se obtiene una baja densidad en el caso de proceso de compactación y sinterización de los polvos, aunque esta aumenta con la temperatura de sinterización, ya que muestra unas porosidades alrededor del 16\% para las temperaturas de 1250 y $1300{ }^{\circ} \mathrm{C}$, que se reduce a un $9 \%$ cuando la sinterización se realiza a $1400{ }^{\circ} \mathrm{C}$. Sin embargo, la densidad obtenida en el proceso SPS resulta muy cercana a la teórica, con porosidades que no alcanzan en ningún caso el 1\% (Tabla 3). Estos resultados se confirman con la caracterización microestructural mediante microscopía óptica (Fig. 3), donde se aprecia la evolución de la porosidad, en el proceso convencional de compactación y sinterización, con la temperatura, y la ausencia prácticamente por completo en las muestras procesadas por SPS.

Mediante la preparación metalográfica de una sección transversal y con el software de tratamiento de imagen (IpWin32) podemos observar la porosidad existente en la muestra. 

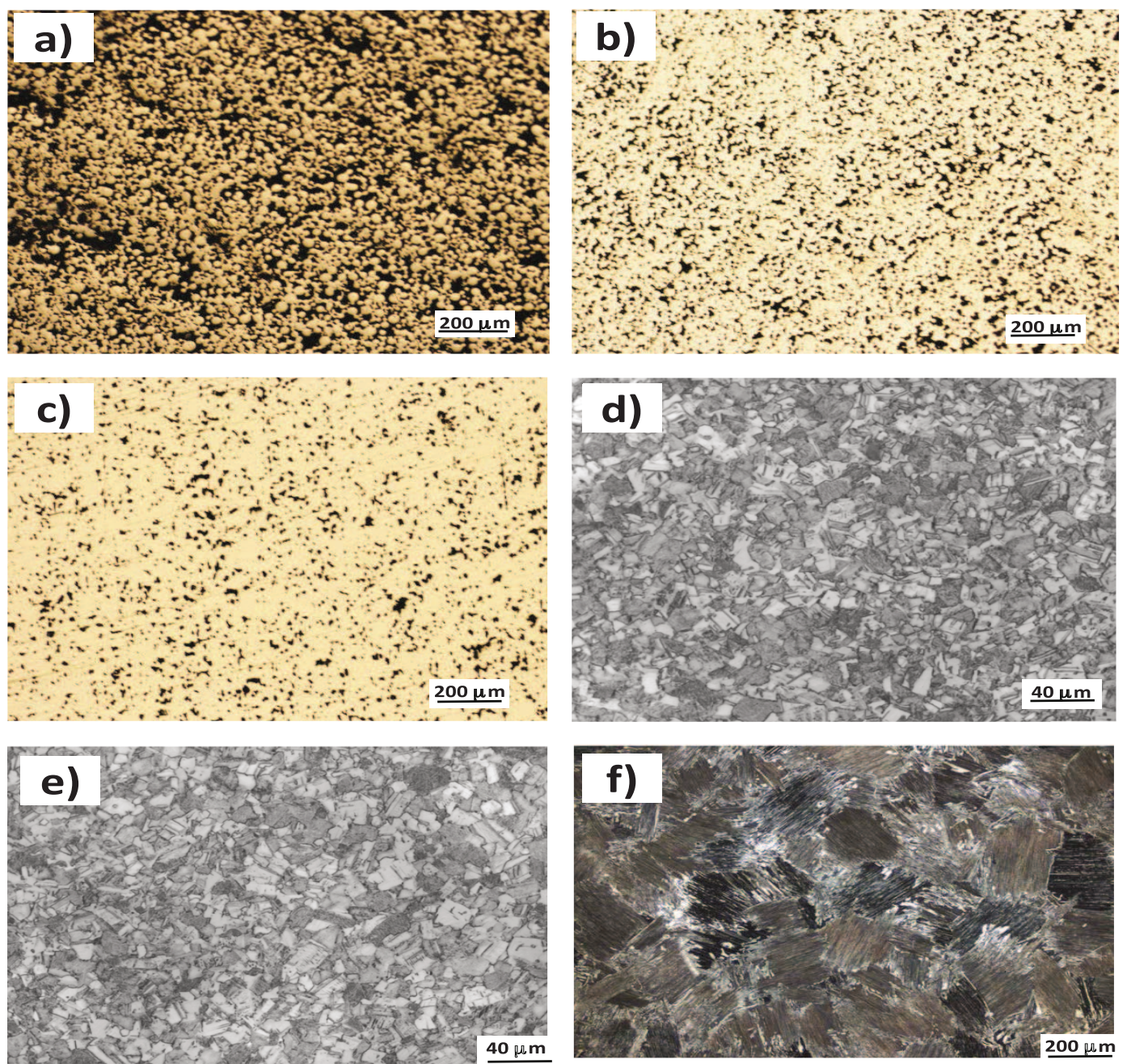

Fig. 3. Imágenes de microscopía óptica de las distintas muestras obtenidas. a) 1-CS, b) 2-CS, c) 3-CS, d) 1-SPS, e) 2-SPS y f) 3-SPS.

Estos resultados también se observan mediante microscopía electrónica de barrido, en cuyas imágenes se aprecian con mayor detalle los problemas de sinterización obtenidos en las muestras compactadas y sinterizadas (Fig. 4a y $4 \mathrm{~b})$, donde se indican mediante flechas las zonas donde aparece una falta de formación de cuellos de sinterización, incluso para temperaturas de $1400{ }^{\circ} \mathbf{C}$. Ello es concordante con las densidades finales obtenidas, que a pesar de su mayor densificación sigue presentado, para esta temperatura, porosidades cercanas al $9 \%$. En su microestructura, observada en las Fig. 4, aparece una tipología dúplex cuando se sinteriza hasta $1300^{\circ} \mathbf{C}$ (Fig. 4a) y una microestructura lamelar cuando la temperatura ya es de $1400{ }^{\circ} \mathrm{C}$ (Fig. $4 b)$; sin embargo, en el proceso SPS se obtiene microestructura dúplex por debajo de los $1300^{\circ} \mathbf{C}$ (Fig. 4c) y microestructura lamelar por encima de esa temperatura (Fig. 4d). 
TABLA 3

DENSIDAD y POROSIDAD DE LAS MUESTRAS OBTENIDAS POR EL MÉTODO DE ARQUÍMEDES

\begin{tabular}{|c|c|c|}
\hline Muestra & $\begin{array}{c}\text { Densidad de Arquímedes (g/ } \\
\left.\mathrm{cm}^{3}\right)\end{array}$ & Porosidad (\%) \\
\hline 1-CS & $3,34( \pm 0.01)$ & 17,41 \\
\hline 2-CS & $3,24( \pm 0.01)$ & 16,70 \\
\hline 3-CS & $3,45( \pm 0.02)$ & 8,93 \\
\hline 1-SPS & $4,01( \pm 0.01)$ & 0,91 \\
\hline 2-SPS & $4,05( \pm 0.02)$ & 0,05 \\
\hline 3-SPS & $4,03( \pm 0.01)$ & 0,03 \\
\hline
\end{tabular}
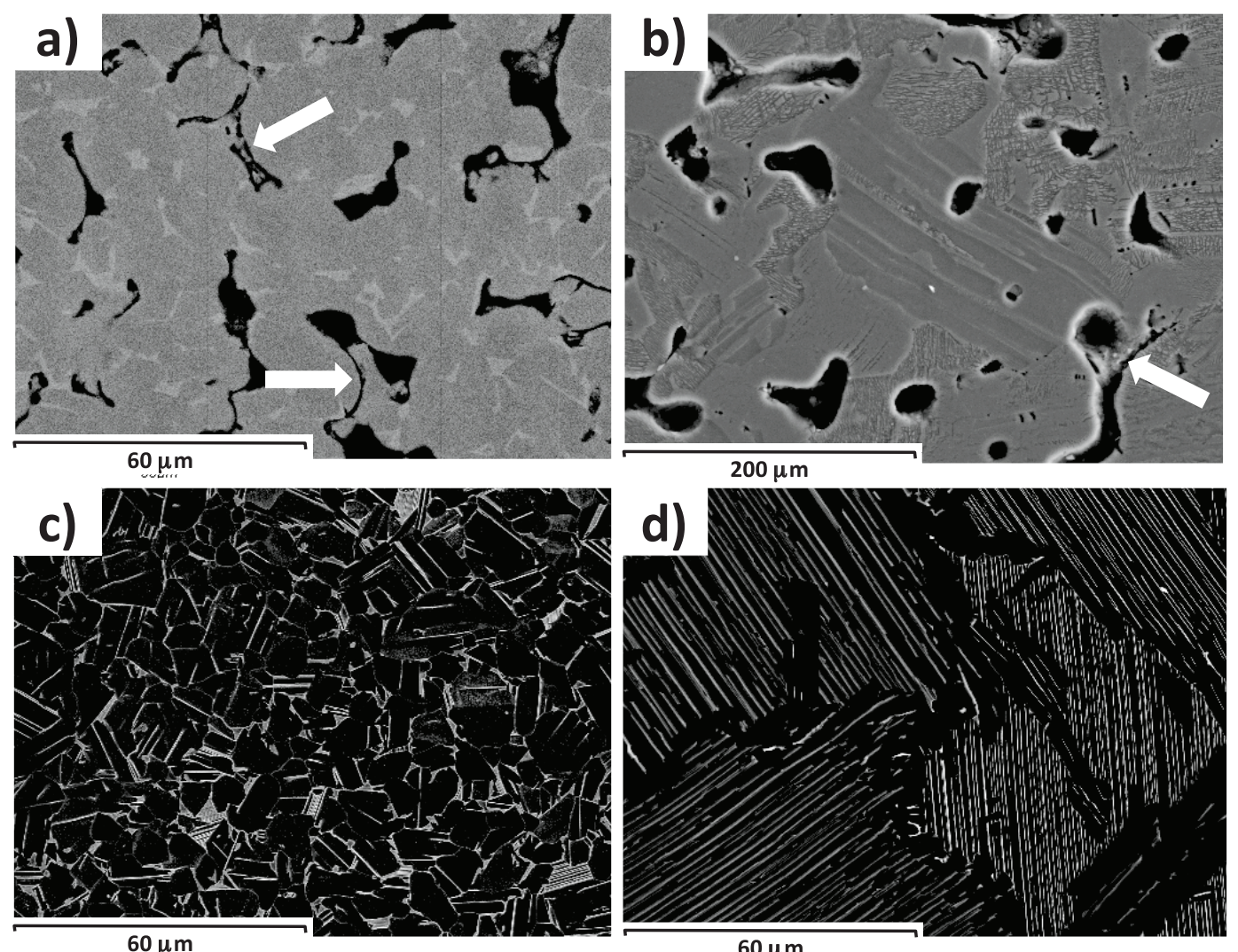

Fig. 4. Imágenes de electrones retrodispersados,

a) muestra 2-CS, b) muestra 2-CS, c) muestra 2-SPS, d) muestra 3-SPS.

Estas microestructuras están formadas por fase $\boldsymbol{\gamma}$-TiAl y $\boldsymbol{\alpha}_{2} \mathrm{Ti}_{3} \mathrm{Al}$. La proporción de fase $\boldsymbol{\alpha}_{2}$ aumenta con la temperatura de sinterización, $\mathrm{y}$ en el procesado por SPS, con el tiempo de permanencia (Tabla 4). Con ello, se dispone de herramientas para obtener la microestructura adecuada en función de las condiciones del proceso. Una mayor temperatura permite 
obtener menor porosidad, una mayor cantidad de transformación de fase $\boldsymbol{\alpha}_{2}$, pero con un aumento del tamaño de grano pasando de microestructura dúplex a lamelar, cuando la temperatura es superior a los $1300^{\circ} \mathbf{C}$.

\section{TABLA 4}

PorCEnTAJE DE FASE $\boldsymbol{\alpha}_{2}$ EN FUNCIÓN DE LA TEMPERATURA DE PROCESADO

\begin{tabular}{|c|c|c|c|}
\hline Muestra & Temperatura $\left({ }^{\mathbf{0}} \mathbf{C}\right)$ & Fase $\gamma \operatorname{TiAl}(\%)$ & Fase $\boldsymbol{\alpha}_{2} \operatorname{Ti}_{3} \mathrm{Al}(\%)$ \\
\hline 1-CS & 1250 & 89,0 & 11,0 \\
\hline 2-CS & 1300 & 87,9 & 12,2 \\
\hline 3-CS & 1400 & 46,4 & 53,6 \\
\hline 1-SPS & 1270 & 85,6 & 14,4 \\
\hline 2-SPS & 1270 & 84,4 & 15,6 \\
\hline 3-SPS & 1340 & 74,6 & 25,4 \\
\hline
\end{tabular}

Estas transformaciones microestructurales se observan en la Fig. 5, en la que se incluyen los microanálisis por energías dispersivas de rayos $\mathrm{X}$ realizados, en los que se aprecian las diferencias de composición entre las dos fases presentes, $\gamma$-TiAl y $\boldsymbol{\alpha}_{2} \mathrm{Ti}_{3} \mathrm{Al}$, con un reparto homogéneo del niobio entre las dos fases y una difusión ligeramente mayor del cromo hacia la fase $\boldsymbol{\alpha}_{2}$ (Fig. 5b). De todos modos debe tenerse en cuenta el efecto de volumen, en el microanálisis, que presenta una importante influencia de los átomos vecinos, a los aumentos a los que se ha realizado el análisis.
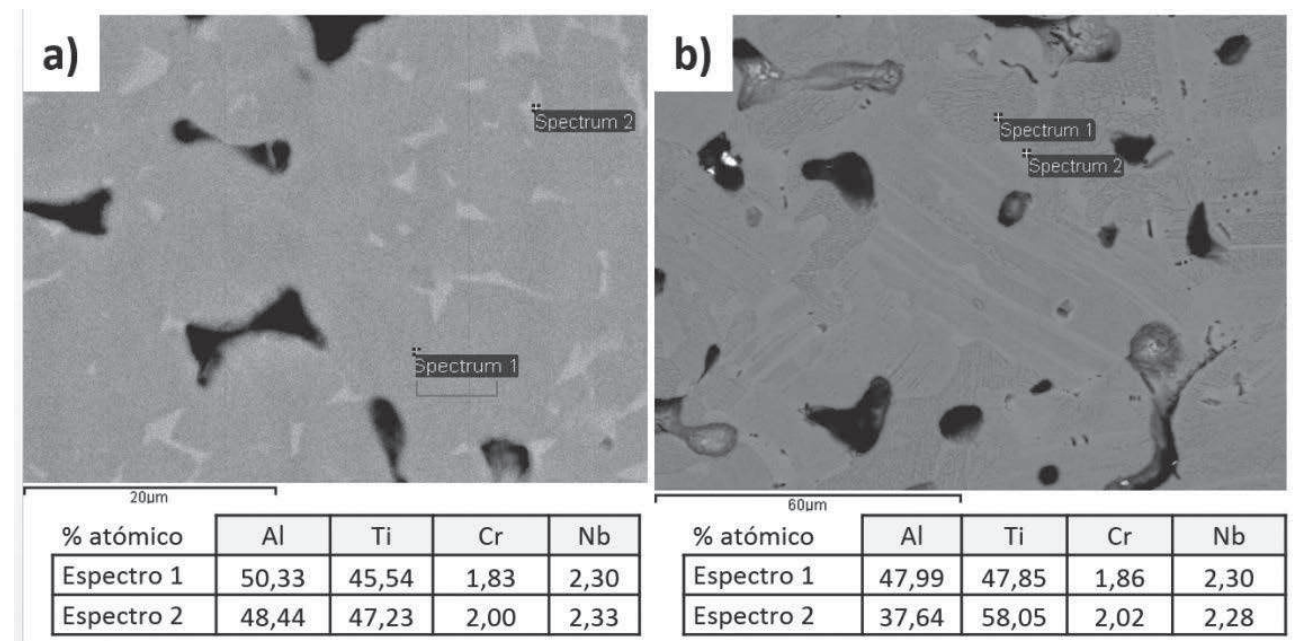

Fig. 5. Detalle de la microestructura obtenidas en: a) sinterizada a $1250{ }^{\circ} \mathrm{C}$, y b) sinterizada a $1400{ }^{\circ} \mathrm{C}$. Se indica los resultados del porcentaje atómico en los espectros de energías dispersivas de rayos $\mathrm{X}$ obtenidos.

De igual modo, se aprecian transformaciones semejantes cuando se procesa el polvo por SPS, con la formación de las estructuras dúplex o lamelares en función de la temperatura (ver Fig.
4 c y d), y microanálisis de las dos fases, $\gamma$ (TiAl) y $\boldsymbol{\alpha}_{2}\left(\mathrm{Ti}_{3} \mathrm{Al}\right)$ semejantes, apreciándose igualmente una distribución uniforme del niobio entre ambas 
fases y una ligera mayor concentración de cromo en la fase $\boldsymbol{\alpha}_{2}$.
El análisis por difracción de rayos X (Fig. 6) nos permite confirmar la presencia de las fases obtenidas en las distintas muestras, principalmente, $\gamma$ (TiAl) y $\boldsymbol{\alpha}_{2}\left(\mathrm{Ti}_{3} \mathrm{Al}\right)$.

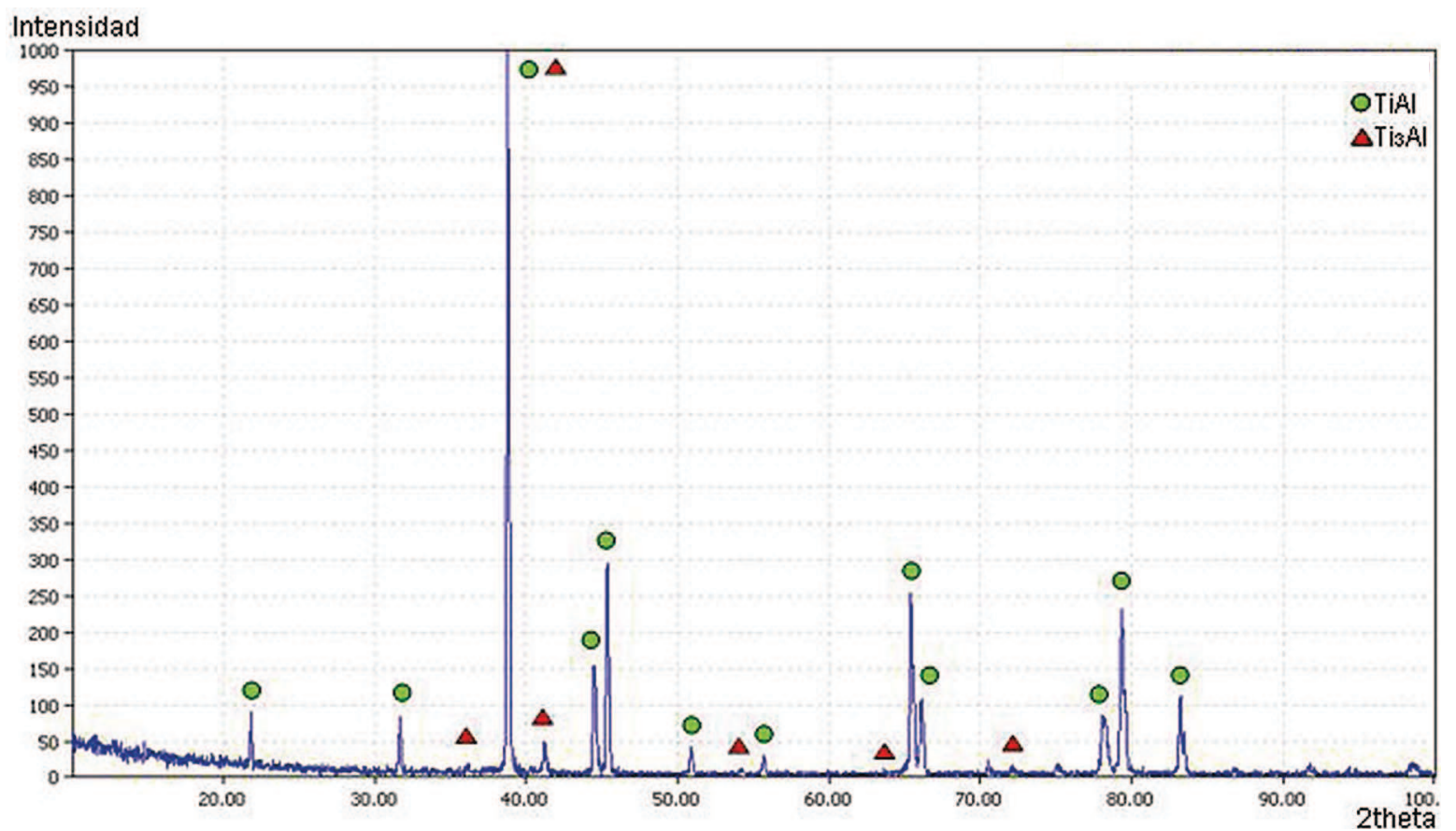

$\mathrm{F}$

Fig. 6. Difractograma de la muestra 2 obtenida por SPS, en el que puede apreciarse la formación de fase $\alpha_{2}$ y la fase mayoritaria $\gamma$.

Esto hace que la dureza de las distintas muestras obtenidas en los dos procesos resulten diferentes por la porosidad implicada en el procesado de compactación + sinterización, frente a la densificación casi total del procesado por SPS. De este modo no ha sido posible obtener la dureza, de los intermetálicos compactados y sinterizados, en una escala que pudiera compararse con la dureza de las muestras obtenidas por SPS. Sin embargo, la microdureza obtenida, en la que se ha tenido especial cuidado en que la porosidad no influya en los resultados obtenidos, presenta unas diferencias atribuibles al efecto térmico envuelto en el proceso, y por ello presenta diferencias por el engrosamiento de grano o la obtención de la microestructura lamelar a elevadas temperaturas (Tabla 5).

La microdureza del polvo, debido a su obtención mediante atomizado, resulta la mayor obtenida, 481,0 HV. En las muestras sinterizadas obtenemos una mayor dureza a medida que aumenta la temperatura de sinterización, 1200 y $1300{ }^{\circ} \mathrm{C}$, con microestructura dúplex, disminuyendo ligeramente, a pesar de la mayor sinterización, cuando la microestructura es lamelar, sinterizada a $1400{ }^{\circ} \mathrm{C}$. Ello viene explicado por la mayor densificación con la temperatura y el aumento, al mismo tiempo, de la proporción de fase $\alpha_{2}$, pero a su vez se produce un aumento del tamaño de grano, lo que disminuiría su dureza; de ahí la semejanza en los resultados obtenidos. 
TABLA 5

MEdidAs DE MICRODUREZA EN LAS DISTINTAS MUESTRAS

\begin{tabular}{|c|c|c|}
\hline Muestra & Parámetros & Microdureza HV \\
\hline Polvo 30 micras & & $481,0 \pm 19,5$ \\
\hline 1-CS & $1250^{\circ} \mathrm{C}, 120$ min. & $276,3 \pm 19,8$ \\
\hline 2-CS & $1300^{\circ} \mathrm{C}, 120$ min. & $343,0 \pm 17,6$ \\
\hline 3-CS & $1400^{\circ} \mathrm{C}, 120$ min. & $319,3 \pm 20,2$ \\
\hline 1-SPS & $1270^{\circ} \mathrm{C}, 2$ min. & $397,3 \pm 20,6$ \\
\hline 2-SPS & $1270^{\circ} \mathrm{C}, 6$ min. & $369,8 \pm 13,4$ \\
\hline 3-SPS & $1340^{\circ} \mathrm{C}, 2$ min. & $378,7 \pm 13,8$ \\
\hline
\end{tabular}

Esta influencia del aumento en el tamaño de grano se confirma en las muestras procesadas por SPS, donde la mayor dureza corresponde a la mínima transformación del polvo original y, por tanto, a las temperaturas más bajas y tiempos de exposición a la temperatura menores. En general, puede apreciarse una mayor microdureza en las muestras SPS que en las compactadas y sinterizadas, precisamente por el mejor control del tamaño de grano y la menor transformación térmica durante el proceso (Tabla 5).

Esto mismo viene a confirmarse en los resultados obtenidos en los ensayos de compresión para las muestras compactadas y sinterizadas (Tabla 6), donde se aprecia un incremento en función de la temperatura; sin embargo, debe señalarse que para las temperaturas de 1250 y $1300{ }^{\circ} \mathrm{C}$ no se aprecia mayor resistencia mecánica a compresión, pero sí una mayor capacidad de deformación por la mayor sinterización con el aumento de la temperatura o, lo que es lo mismo, una menor porosidad. Para las mayores temperaturas, la resistencia incrementa en un $45 \%$ al combinarse la menor porosidad (véase la Tabla 3) con la formación de la microestructura lamelar. No obstante, estos resultados quedan lejos de los obtenidos por Y. Huang y colaboradores [19].

TABLA 6

PROPIEDADES A COMPRESIÓN DE LAS MUESTRAS COMPACTADAS Y SINTERIZADAS A DIFERENTES TEMPERATURAS

\begin{tabular}{|c|c|c|c|}
\hline Muestra & Temperatura $\left({ }^{\circ} \mathbf{C}\right)$ & $\boldsymbol{\sigma}_{\max }(\mathrm{MPa})$ & $\boldsymbol{\varepsilon}_{\max }(\mathrm{mm} / \mathrm{mm})$ \\
\hline 1-CS & 1250 & $569,2( \pm 0.1)$ & 0,252 \\
\hline 2-CS & 1300 & $577,9( \pm 0.1)$ & 0,344 \\
\hline 3-CS & 1400 & $829,8( \pm 0.1)$ & 0,235 \\
\hline
\end{tabular}

En la tabla no se indica el valor de la rigidez, pues en todos los casos se ha producido rotura catastrófica con parcial desmoronamiento del polvo, debido a su elevada porosidad, y, por tanto, los valores medios obtenidos del módulo de elasticidad de $33 \mathrm{GPa}$ resultan muy inferiores a los obtenidos por otros autores, que se encuentran entre 72 y $180 \mathrm{GPa}[8]$.

De los ensayos de flexión a 3 puntos, realizados con el material sinterizado a $1300{ }^{\circ} \mathrm{C}$, se extraen, en primer lugar, evidencias de la poca 
sinterización, ya que al hacer el ensayo por ultrasonidos encontramos fisuras en dos de ellos, los cuales quedan descartados para el ensayo de flexión.

La resistencia máxima a flexión obtenida es de $215 \mathrm{MPa}$, lo que responde a resultados aceptables si se compara con estudios de TiAl obtenido mediante SPS, donde los valores obtenidos cuando la sinterización no es completa oscilan entre los 254 y los $442 \mathrm{MPa}$ [20]. Pero lo que resulta especialmente problemático es la baja plasticidad que apenas alcanza el $2 \%$, y ello para las temperaturas que proporcionan la máxima densificación.

Este conjunto, elevada porosidad y baja ductilidad, provoca una fractura frágil transgranular característica, tal como puede apreciarse en la Fig. 7a, correspondiente a la fractura de la muestra sinterizada a $1300^{\circ} \mathrm{C}$, en la que se aprecia la baja sinterización obtenida y la escasa superficie de fractura que explicaría la baja ductilidad a pesar de contar con una resistencia a temperatura ambiente, solo ligeramente inferior a la obtenida por otras técnicas.

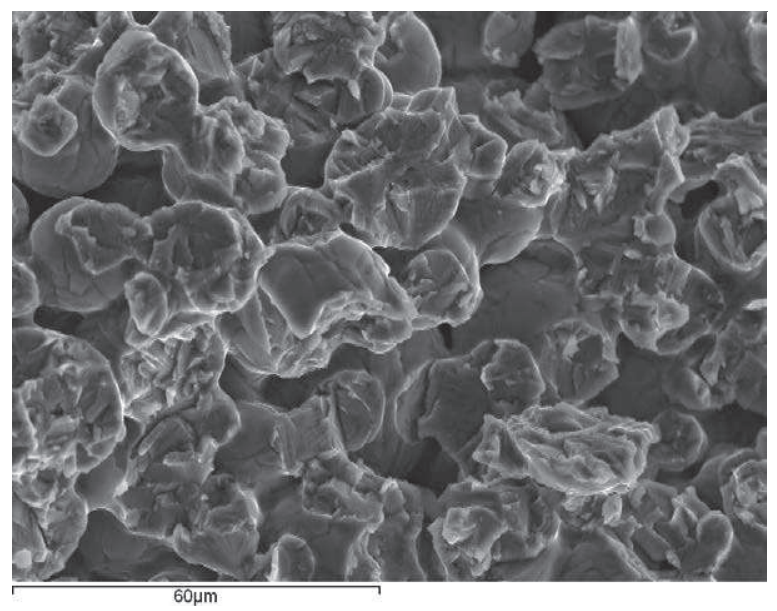

a)

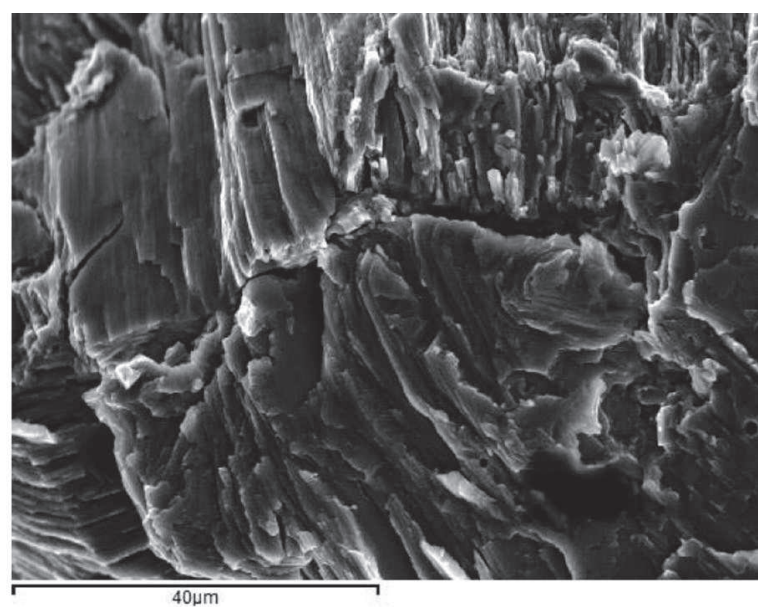

b)

Fig. 7. Imagen de electrones secundarios de la superficie de fractura a flexión: a) muestra sinterizada a $1300{ }^{\circ} \mathbf{C}$, b) muestra SPS procesada a $1340^{\circ} \mathrm{C}$ durante 2 minutos.

En las muestras procesadas mediante SPS, especialmente la procesada a mayor temperatura, que presenta la microestructura lamelar (Fig. 7b), se aprecia una fractura frágil transgranular con desviación de la fisura principal [21], que llega a proporcionar una ductilidad cercana al 5\%, con resistencias máximas que alcanzan los $960 \mathrm{MPa}$.

\section{Conclusiones}

De los resultados obtenidos puede indicarse que es posible obtener productos de intermetálicos TiAl mediante las técnicas de pulvimetalurgia convencionales, pero la sinterización requiere de elevadas temperaturas y largos tiempos de permanencia, y de todos modos sigue mostrando una elevada porosidad, cercana al $9 \%$, en el mejor de los casos, y temperaturas de $1400{ }^{\circ} \mathbf{C}$. Por el contrario, el procesado por SPS proporciona una excelente densificación y propiedades adecuadas en el producto, lo que hace que sea un proceso de conformación muy esperanzador para este tipo de intermetálicos.

La microestructura obtenida a temperaturas inferiores a $1300{ }^{\circ} \mathrm{C}$ es dúplex, con formación 
de pequeñas cantidades (alrededor del 10-12\%) de fase $\alpha_{2}\left(\mathrm{Ti}_{3} \mathrm{Al}\right)$ para las muestras compactadas y sinterizadas, y algo mayor, alrededor del $15 \%$, para las muestras procesadas por SPS. Sin embargo, a temperaturas más elevadas, 1400 ${ }^{\circ} \mathbf{C}$, la compactación y sinterización proporciona estructuras lamelares con más del $50 \%$ de fase $\alpha_{2}$, mientras tan solo alcanza el $25 \%$ para las muestras procesadas por SPS.

En todas las muestras aparecen dos fases, $\gamma+$ $\alpha_{2}$, que corresponden a las estructuras cristalinas tetragonal, centrada en las caras, y hexagonal, respectivamente, confirmado por los análisis de difracción de rayos $\mathrm{X}$. En ellas, el niobio se reparte de forma homogénea, mientras que el cromo tiende a formar parte, de forma preferente, de la fase $\alpha_{2}$ de $\mathrm{Ti}_{3} \mathrm{Al}$.

Las propiedades mecánicas de las muestras compactadas y sinterizadas aumentan con la temperatura de sinterización, tanto en la microdureza como en el ensayo de compresión, aunque estas propiedades continúan siendo bajas, en todo caso, por la baja sinterización del proceso. Esto se confirma igualmente en el ensayo de flexión que presenta resistencias máximas del orden de los $215 \mathrm{MPa}$, muy inferiores a los 960 $\mathrm{MPa}$ obtenidos en las muestras procesadas por SPS.

Esta mejora en las propiedades mecánicas de las muestras procesadas mediante SPS viene justificadas por el mayor control del tamaño de grano, pues el tiempo de permanencia a elevadas temperaturas es muy pequeño, 2 y 6 minutos, presentando un ligero aumento del tamaño de grano en función del tiempo del procesado.

\section{Agradecimientos}

Los autores agradecen al Ministerio de Economía y Competitividad del Gobierno de España la financiación recibida a través del proyecto de investigación MAT2011-28492-C03, y a la Generalitat Valenciana (ACOMP/2013/114).

\section{REFERENCIAS}

[1] C. Leyens and M. Peters, Titanium and Titanium Alloy, New York, NY, USA: Wiley-VCH, 2003.

[2] M. Yamaguchi, H. Inuiand and K. Ito, "Hightemperature structural intermetallics", Acta Mater. vol. 48, pp. 307-322, 2000.

[3] W. Xinhua, "Review of alloy process development of TiAl alloys", Intermetallics, vol. 14, pp. 1114-1122, 2006.

[4] T. Kawabata, H. Fukai and O. Izumi, "Effect of ternary additions on mechanical properties of TiAl", Acta Mater. vol. 46, n 6, pp. 2185-2194, 1998.

[5] Z. Chungen, X. Huibin and Y-K. Kyoo, "The influence of additions of $\mathrm{Nb}$ and $\mathrm{Cr}$ on the aluminizing behavior of TiAl alloy", Metall. Mater. Trans. A, vol. 31, pp. 23912394, 2000.

[6] Y. Y. Chen et al., "Effect of spark plasma sintering temperature on microsturcture and mechanical properties of an ultrafine grained TiAl intermetallic alloy", Mat. Sci. Eng. A, vol. 525, pp. 166-175, 2009.

[7] S. L. Xiao et al., "Microstructure end mechanical properties of TiAl alloy prepared by spark plasma sintering". Trans. Nonferrous Met. Soc. China, vol. 19, pp. 1423-1427, 2009.

[8] S. Biamino et al., "Electron beam melting of Ti-48Al-2Cr-2Nb alloy: Microstructure and mechanical properties investigation", Intermetallics, vol. 19, pp. 776-781, 2011. 
[9] A. Sankaran et al., "Texture and microstructure evolution during tempering of gamma-massive phase in a TiAl-based alloy", Intermetallics, vol. 17, pp. 10071016, 2009.

[10] H. Zhu, D.Y. Seo and K. Maruyama, "Strengthening behavior of Beta Phase in Lamellar Microstructure of TiAl Alloys" JOM, vol. 62, n 1, pp. 64-69, 2010.

[11] J.H. Moll and B.J. McTiernan, "PM TiAl alloys: the sky's the limit", MPR, 55 (1), 18-22, 2000

[12] B. Liu et al., "Hot deformation behavior of TiAl alloys prepared by blended elemental powders". Intermetallics, vol. 19, $\mathrm{n}^{\mathrm{o}} 2, \mathrm{pp}$. 154-159, 2011.

[13] I. Agote et al., "Microstructure and mechanical properties of gamma TiAl based alloys produced by combustion synthesis +compaction route", Intermetallics, vol. 16, no 11-12, pp. 1310-1316, 2008.

[14] H. Jabbar et al., "Identification of microstructural mechanisms during densification of a TiAl alloy by spark plasma sintering". J. Alloys Compd., vol. 509, pp. 9826-9835, 2011.
[15] M. A. Lagos and I. Agote, "SPS synthesis and consolidation of TiAl alloys from elemental powders: Microstructure evolution", Intermetallics, vol. 36, pp. 5156, 2013.

[16] H. Wu et al., "Fabrication of TiAl intermetallic by Spark Plasma Sintering", Key Eng. Mater., vol. 336-338, pp. 10501052, 2007.

[17] X. Luetal., "Microstructure and mechanical properties of a spark plasma sintered Ti$45 \mathrm{Al}-8.5 \mathrm{Nb}-0.2 \mathrm{~W}-0.2 \mathrm{~B}-0.1 \mathrm{Y}$ alloy", Intermetallics, vol. 17, pp. 840-846, 2009.

[18] J. L. Murray, en ASM Handbook vol. 3. Alloy Phase Diagrams. ASM International, Ohio. USA. Pag. 2.54. 992, 1987.

[19] Y. Huang et al., "A TiAl based alloy with excellent mechanical performance prepared by gas atomization and spark plasma sintering", Intermetallics, vol. 31, pp. 202207, 2012.

[20] A. Amigó, Estudio preliminar reológico de intermetálicosTiAl obtenidos por SPS. Proyecto fin de carrera, ETSIA de la UPM, Madrid, septiembre 2012.

[21] R. Cao et al., "Fracture behaviour of a TiAl alloy under various loading modes". Eng. Fract. Mech., vol. 75, pp. 4343-4362, 2008. 